\title{
Effective Actions with the First Order Form of Gauge Theories
}

\author{
F. T. Brandt* ${ }^{*}$ \\ Instituto de Física \\ Universidade de São Paulo \\ E-mail: fbrandteusp.br \\ D. G. C. McKeon \\ Department of Applied Mathematics \\ The University of Western Ontario, London, Canada \\ E-mail: dgmckeo2@uwo.ca
}

\begin{abstract}
The first order form of the Einstein-Hilbert action is shown to reduce the number of vertices needed to compute Feynman diagrams to just three three-point ones; in addition there are two propagating fields. This simplified set of Feynman rules can be used to derive the same results as the usual second order form of the Einstein-Hilbert action. We also consider the contributions to the effective action of the graviton in a thermal background.
\end{abstract}

38th International Conference on High Energy Physics

August 3-10, 2016

Chicago, USA

\footnotetext{
* Speaker.

${ }^{\dagger}$ We thank CNPq and Fapesp (Brazil) for financial support.
} 


\section{Introduction}

In the Einstein-Hilbert action

$$
S=\int d^{d} x \sqrt{-g} g^{\mu v} R_{\mu v}(\Gamma),
$$

where

$$
\Gamma_{\mu \nu}^{\lambda}=\frac{1}{2} g^{\lambda \sigma}\left(g_{\mu \sigma, v}+g_{v \sigma, \mu}-g_{\mu \nu, \sigma}\right)
$$

and

$$
R_{\mu v}(\Gamma)=\Gamma_{\mu \rho, v}^{\rho}-\Gamma_{\mu v, \rho}^{\rho}-\Gamma_{\mu \nu}^{\sigma} \Gamma_{\sigma \rho}^{\rho}+\Gamma_{\mu \sigma}^{\rho} \Gamma_{v \rho}^{\sigma}
$$

it is possible to treat both $g_{\mu \nu}$ and $\Gamma_{\mu \nu}^{\lambda}$ as being independent ${ }^{1}$; the equation of motion for $\Gamma_{\mu \nu}^{\lambda}$ in this first order action yields Eq. (1.2).

Employing the first order Einstein-Hilbert (1EH) has the advantage over the usual second order form of the action that the interaction vertices are greatly simplified $[2,3]$. This approach has been employed in the case of the Yang-Mills theory, where it is easy to show that the Green's functions derived from the 1YM and 2YM actions are equivalent. However, it is not readily apparent that the 1EH and 2EH actions lead to the same Green's functions [3]. More recently, we have demonstrated that in fact they are the same [4]. In doing so, we arrive at a set of Feynman rules from the 1EH action that are much simpler than those that follow from the $2 \mathrm{EH}$ action as there are but three three-point vertices and two propagators. We also consider some explicit calculations both at zero and finite temperature which demonstrate the equivalence of the two formulations.

\section{Action and Feynman rules}

It is convenient to use $h^{\mu v}=\sqrt{-g} g^{\mu \nu}$ and $G_{\mu v}^{\lambda}=\Gamma_{\mu \nu}^{\lambda}-\frac{1}{2}\left(\delta_{\mu}^{\lambda} \Gamma_{v \sigma}^{\sigma}+\delta_{v}^{\lambda} \Gamma_{\mu \sigma}^{\sigma}\right)$ as independent fields, so that Eq. (1.1) yields

$$
\mathcal{L}_{1 E H}=h^{\mu \nu}\left(G_{\mu \nu, \lambda}^{\lambda}+\frac{1}{d-1} G_{\mu \lambda}^{\lambda} G_{v \sigma}^{\sigma}-G_{\mu \sigma}^{\lambda} G_{v \lambda}^{\sigma}\right) .
$$

Employing the Faddeev-Popov path integral [5]

$$
Z_{1 E H}=\int \mathcal{D} h^{\mu v} \mathcal{D} G_{\mu v}^{\lambda} \Delta_{F P}(h) \exp i \int d^{d} x\left[\mathcal{L}_{1 E H}+\mathcal{L}_{g f}\right]
$$

and using $h^{\mu v}(x)=\eta^{\mu v}+\phi^{\mu v}(x)$, the propagators $\langle\phi \phi\rangle,\langle G G\rangle,\langle\phi G\rangle$ and the vertex $\langle\phi G G\rangle$ have been obtained in ref. [3]. Subsequently we have proved the equivalence with the usual second order formalism and we have also redefined the fields in such a way that only simple propagators (not mixed) arises [4]. The corresponding generating functional obtained from Eq. (2.2) is given by

$$
\begin{aligned}
Z_{1 E H}= & \int \mathcal{D} h^{\mu v} \mathcal{D} G_{\mu \nu}^{\lambda} \Delta_{F P}(h) \exp i \int d^{d} x\left[\frac{1}{2} G_{\mu v}^{\lambda} M_{\lambda \sigma}^{\mu v} \pi \tau(\eta) G_{\pi \tau}^{\sigma}-\frac{1}{2} \phi_{, \lambda}^{\mu v} M_{\mu v \pi \tau}^{-1 \lambda \sigma}(\eta) \phi_{, \sigma}^{\pi \tau}\right. \\
& \left.+\frac{1}{2}\left(G_{\mu \nu}^{\lambda}+\phi_{, \rho}^{\alpha \beta}\left(M^{-1}\right)_{\alpha \beta \mu \nu}^{\rho \lambda}(\eta)\right)\left(M_{\lambda \sigma}^{\mu v} \pi \tau(\phi)\right)\left(G_{\pi \tau}^{\sigma}+\left(M^{-1}\right)_{\pi \tau \gamma \delta}^{\sigma} \underset{\xi}{\xi}(\eta) \phi_{, \zeta}^{\gamma \delta}\right)+\mathcal{L}_{g f}\right],
\end{aligned}
$$

\footnotetext{
${ }^{1}$ This form of the action is often credited to Palatini, though Einstein first considered it [1]
} 
where $M_{\lambda \sigma}^{\mu v} \pi \tau(h)$ is a simple tensor given by Eq. (3.6) of ref. [4], so that there is two simple propagators $\langle\phi \phi\rangle$ and $\langle G G\rangle$, the usual ghost propagator and four interaction vertices. This is a quite remarkable result when compared with the infinite number of interaction vertices which arises in the usual second order formalism. Furthermore, these Feynman rules are expressed in a much more compact form (see Eqs. (3.25) of ref. [4]) in terms of $M_{\lambda}^{\mu v} \pi \tau$ and its inverse.

\section{Thermal effective action}

Using the Feynman rules in the first order formalism and the thermal field theory imaginary time formalism we obtain the following result for the thermal one-graviton function

$$
\Gamma_{\mu \nu}^{\mathrm{th}}=\frac{d(d-3)}{2} \frac{\zeta(d) \Gamma(d)}{2(d-1)} \frac{2 \pi^{\frac{d-1}{2}}}{\Gamma\left(\frac{d-1}{2}\right)} \frac{T^{d}}{(2 \pi)^{d-1}}\left(\eta_{\mu \nu}-d u_{\mu} u_{v}\right),
$$

where $u$ is the heat bath four velocity, $\zeta$ is the Riemann zeta function and $\Gamma$ is the gamma function. The factor $d(d-3) / 2$ counts the number of degrees of freedom of the graviton field in $d$ dimensions. For $d=4$

$$
\left.\Gamma_{\mu v}^{\mathrm{th}}\right|_{d=4}=\frac{\pi^{2} T^{4}}{90}\left(\eta_{\mu v}-4 u_{\mu} u_{v}\right),
$$

which is in agreement with the known result obtained using the second order formalism [7]. The calculation of higher order contributions to the thermal effective action, which includes contributions from all the $n$-graviton thermal Green functions, is work in progress.

\section{References}

[1] M. Ferraris, M. Francaviglia, and C. Reina, Gen. Rel. Grav. 14, 243 (1982).

[2] D. G. C. McKeon, Can. J. Phys. 72, 601 (1994).

[3] F. T. Brandt and D. G. C. McKeon, Phys. Rev. D 91, 105006 (2015).

[4] F. T. Brandt and D. G. C. McKeon, Phys. Rev. D 93, 105037 (2016).

[5] L. D. Faddeev and V. N. Popov, Phys. Lett. B25, 29 (1967).

[6] G. 't Hooft and M. J. G. Veltman, Annales Poincare Phys. Theor. A20, 69 (1974).

[7] P. S. Gribosky, J. F. Donoghue, and B. R. Holstein, Annals Phys. 190, 149 (1989); A. Rebhan, Nucl. Phys. B351, 706 (1991); F. T. Brandt and J. Frenkel, Phys. Rev. D58, 085012 (1998). 